\title{
Professor Dr. med. habil. Joachim Schauer zum 70. Geburtstag
}

\author{
Professor Dr. med. habil. Joachim Schauer on the Occasion of his 70th Birthday
}

\section{Bibliografie}

DOI 10.1055/s-0029-1215160

Pneumologie 2009; 63: 594-595 @ Georg Thieme Verlag KG Stuttgart · New York ISSN 0934-8387

Korrespondenzadresse PD Dr. med. Gerhard Hoheisel August-Bebel-Str. 69 04275 Leipzig gerhard.hoheisel@t-online.de

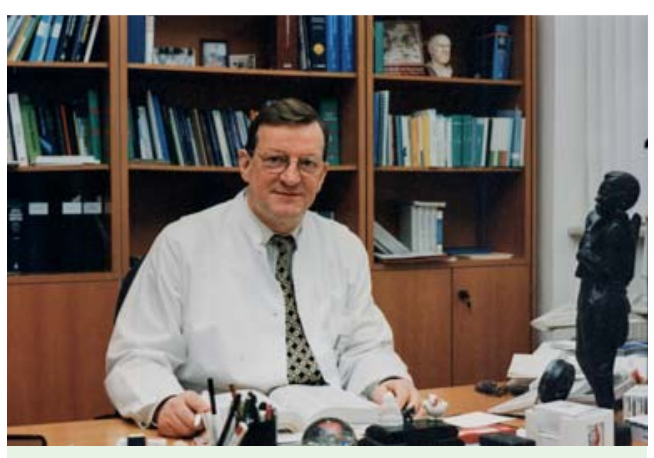

Abb. 1 Prof. Schauer in seinem Arbeitszimmer.

Am 25. April 2009 vollendete Herr Professor Schauer, der frühere Leiter der Medizinischen Klinik I der Universität Leipzig, das 70. Lebensjahr (৫ Abb. 1).

In Magdeburg geboren, studierte er an der altehrwürdigen Universität Leipzig - die in diesem Jahr 600 Jahre alt wird - Medizin. Sein Staatsexamen legte er 1963 ab und sammelte als Assistenzarzt im Bezirkskrankenhaus Grimma seine ersten internistischen Erfahrungen. Noch im selben Jahr promovierte er zum Thema „Umgebungsuntersuchungen bei Hepatitis infectiosa“, was für sein späteres Interesse jedoch nicht richtungsweisend war. Zurück in Leipzig habilitierte er sich 1976 an der damaligen „Karl-Marx-Universität“ mit dem Thema „Diagnostik der gestörten kardiopulmonalen Funktion bei der ischämischen Herzkrankheit".

Vom Herzen her kommend war er also bei der Lunge angelangt. Früh etablierte er in Leipzig die Einschwemmkatheter-Untersuchung des rechten Herzens. So wurde ihm 1983 wegen seiner fachlichen Qualifikation die Leitung der Abteilung Pneumologie der Medizinischen Klinik übertragen, die er in gemeinsamem Enthusiasmus mit seinen Mitarbeiterinnen und Mitarbeitern trotz bescheidener Mittel aufbaute. 1987 wurde er erster Stellvertreter des Direktors der Klinik für In- nere Medizin, 1988 außerordentlicher, 1990 nach der Wende ordentlicher Professor der Universität Leipzig und demokratisch gewählter (!) Direktor der Klinik für Innere Medizin ( $\bullet$ Abb. 2). Als Prodekan konnte er gemeinsam mit dem damaligen Dekan Professor Geiler und weiteren Persönlichkeiten entscheidend an der akademischen Neugestaltung der Medizinischen Fakultät mitwirken. In der Sächsischen Gesellschaft für Innere Medizin war er Vorstandsmitglied, Vorsitzender und wiederholt Tagungspräsident. Als Leitender Ärztlicher Direktor des Klinikums und Medizinischer Vorstand half er, die Neugestaltung der Organisationsstrukturen des Universitätsklinikums Leipzig einzuleiten. Auch seinem engagierten Einsatz in verschiedenen Gremien, nicht zuletzt bei der Staatsregierung in Dresden, ist der gelungene Neubau des Universitätsklinik-Campus Leipzig mitzuverdanken.

Professor Schauer hat den Grundstein zu einer anerkannten, selbstständigen universitären Pneumologie in Leipzig gelegt, an deren Ausbau er kontinuierlich gewirkt hat. Er förderte seine „jungen Leute“, wo er nur konnte, und ermöglichte es ihnen, auch nationale und internationale Erfahrung zu sammeln ( $\bullet$ Abb. 3). Ein Höhepunkt seiner Karriere war die gelungene Gestaltung der 39. Jahrestagung der Deutschen Gesellschaft für Pneumologie (DGP) 1998 in Leipzig, die erste Jahrestagung in einem der neuen Bundesländer (৫ Abb. 4).

Von Anfang an befürwortete er die integrative Sicht auf kardiopulmonale Erkrankungen. Er etablierte ein Zentrum für Patienten mit pulmonaler Hypertonie, welches seither besteht. Früh setzte er sich auch für die Etablierung der Lungentransplantation gemeinsam mit dem Herzzentrum der Universität Leipzig ein. Auch erkannte er die Notwendigkeit einer Spezialsprechstunde für die erwachsenen Patienten mit Mukoviszidose sowie eines kompletten Schlaflabors. Für beides schuf er die Voraussetzungen. 


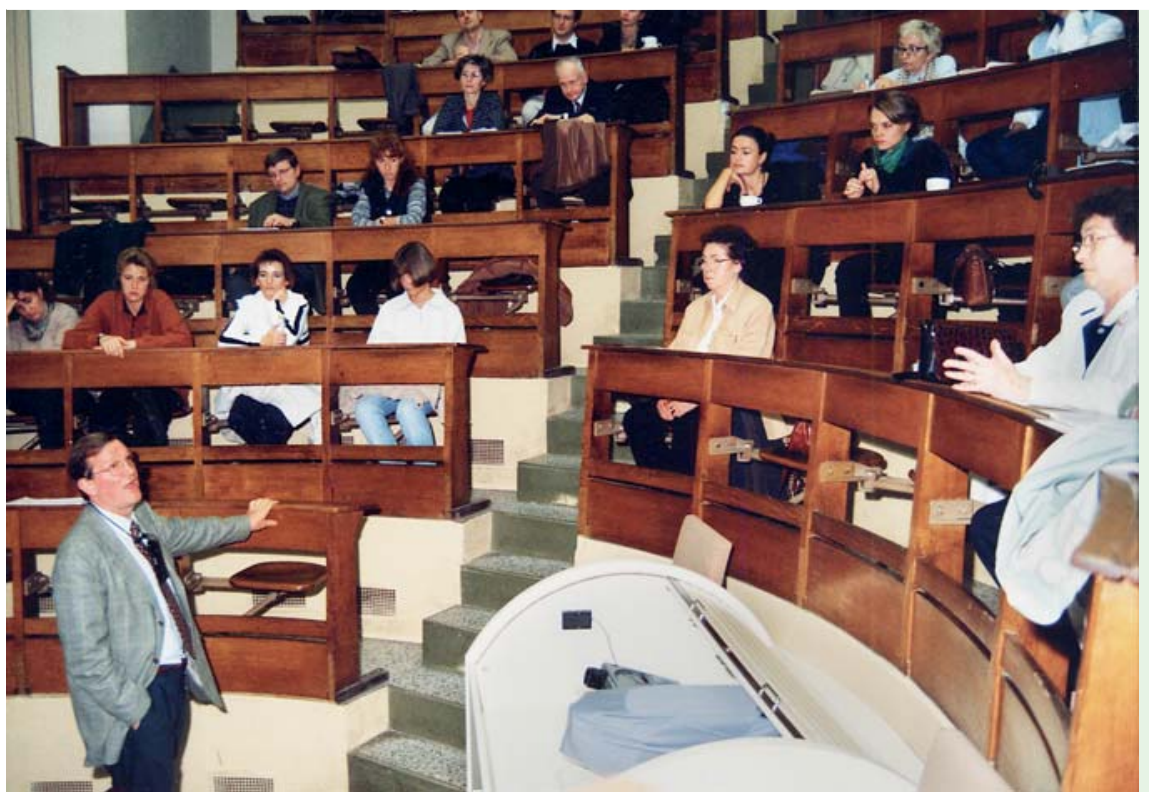

Abb. 2 Vorlesung im alten Hörsaal des Zentrums für Innere Medizin.

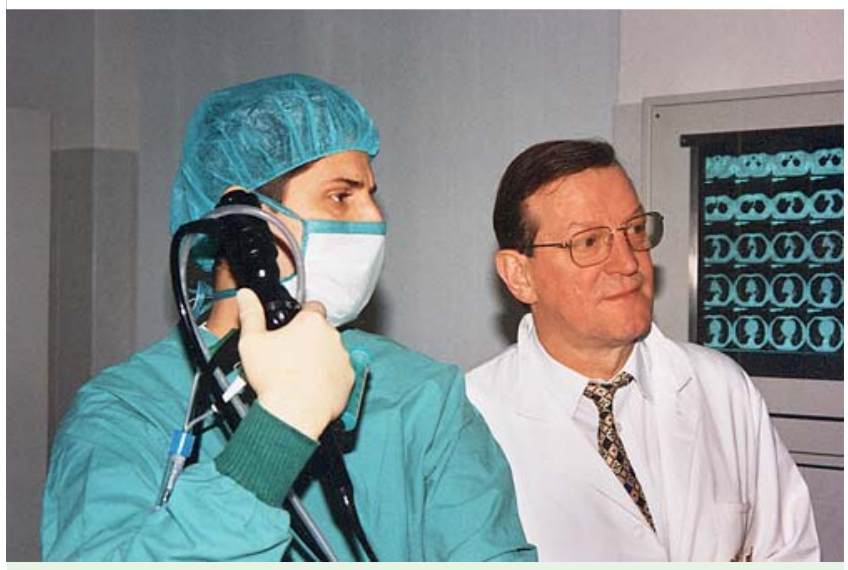

Abb. 3 Bei der Bronchoskopie mit J. Winkler.

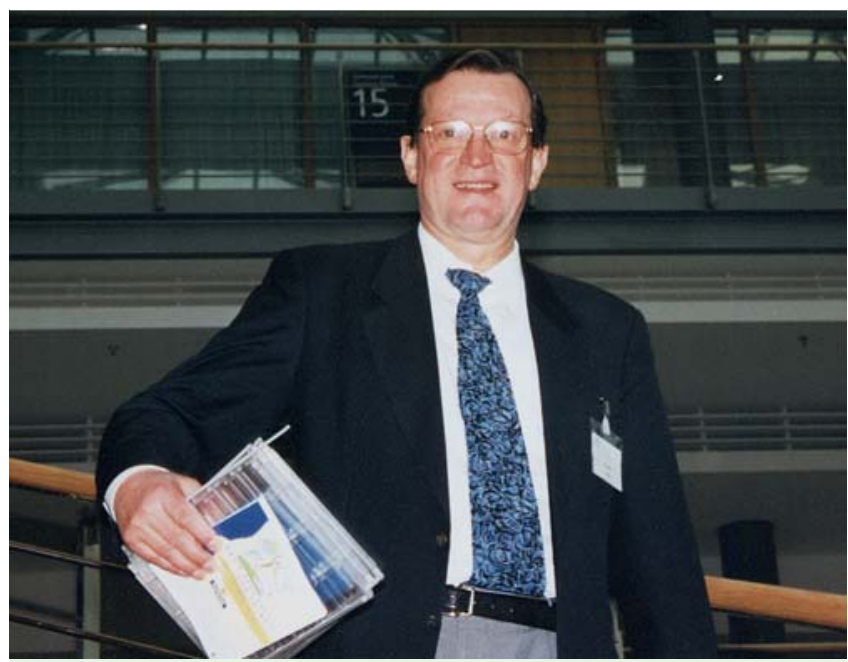

Abb. 4 DGP-Kongresspräsident 1998 in Leipzig.

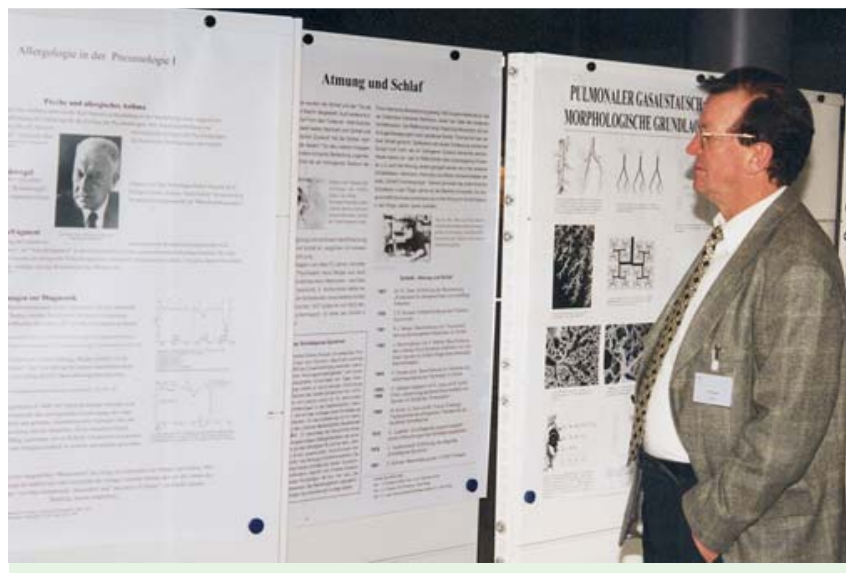

Abb. 5 Am Poster.

Besondere Weitsicht bewies er mit der von ihm wesentlich betriebenen Initiative zur Gründung der Mitteldeutschen Gesellschaft für Pneumologie, die in den Bundesländern Sachsen, Sachsen-Anhalt und Thüringen lebendig tätig ist, geschätzt wird und ein bemerkenswertes Beispiel der Kooperation zwischen Pneumologen in Kliniken und Niederlassung darstellt ( Abb.5).

Nach dieser dynamischen Zeit fiel ihm der Gedanke an einen Ruhestand nicht leicht. So war er zunächst auch noch klinisch, später im niedergelassenen Bereich sehr aktiv. Gutachtertätigkeit führt er bis heute noch aus. Professor Schauer ist weiterhin ein sehr geschätzter Berater und pragmatischer Hochschullehrer mit vielen guten Verbindungen, auf dessen fachliche und menschliche Qualifikation wir uns gerne verlassen.

Professor Schauer beeindruckt immer wieder mit seinem exzellenten Wissen, was Literatur, Geschichte und insbesondere die Musik betrifft. Besonderes Interesse gilt den Komponisten Richard Wagner und Gustav Mahler. Es ist eine Freude, mit Joachim Schauer über die Geschichte zu diskutieren oder von der Musik zu schwärmen. Wir wünschen ihm viele Jahre in guter Gesundheit, Schaffenskraft und Lebensfreude gemeinsam mit seiner Ehefrau Christel, seinen Kindern Ulrike und Christof, seinen Enkelkindern, Freunden und Kollegen. 